\title{
Criminologie
}

\section{À propos de l'offre de traitement en milieu correctionnel : guérir, soigner, aider}

\section{Dianne Casoni}

Volume 29, numéro 1, printemps 1996

Intervenir auprès des délinquants

URI : https://id.erudit.org/iderudit/017383ar

DOI : https://doi.org/10.7202/017383ar

Aller au sommaire du numéro

Éditeur(s)

Les Presses de l'Université de Montréal

ISSN

0316-0041 (imprimé)

1492-1367 (numérique)

Découvrir la revue

Citer cet article

Casoni, D. (1996). À propos de l'offre de traitement en milieu correctionnel : guérir, soigner, aider. Criminologie, 29(1), 109-120.

https://doi.org/10.7202/017383ar
Résumé de l'article

This paper adresses the complex unconscious identificatory processes which may silently be active within the clinician when he proposes treatment either to a child or adolescent in an institution or to an adult in a correctionnal setting. Three representations of treatment serve as a backdrop to analyse different identificatory movements on the part of the clinician and how such unconscious motivations may affect his work. 
This paper adresses the complex unconscious identificatory processes which may silently be active within the clinician when he proposes treatment either to a child or adolescent in an institution or to an adult in a correctionnal setting. Three representations of treatment serve as a backdrop to analyse different identificatory movements on the part of the clinician and how such unconscious motivations may affect his work.

\section{Black bird singing in the dead of night take these sunken eyes and learn to see all your life you were only waiting for this moment to be free. \\ (John Lennon et Paul McCartney, 1968)}

Le problème éthique posé par le traitement en milieu carcéral ou rééducatif a déjà été examiné avec intelligence au Québec. Citons pour mémoire le texte de Bertrand (1981) qui, en s'appuyant sur le philosophe John Stuart Mill (1859), souligne la nécessité de distinguer le sujet demandant de l'aide du sujet chez qui cette demande est absente afin de ne pas transformer l'intervention en une nouvelle expérience de contrainte.

Le propos que nous aimerions développer dans cet essai vise cependant un autre but et cherchera à proposer une réflexion originant non pas de la perspective du sujet mais de l'offre de service du criminologue, psychologue, éducateur ou autre intervenant clinique œuvrant en milieu correctionnel ou rééducatif. Nous chercherons ainsi à interroger quelles peuvent être les motivations inconscientes chez l'intervenant qui sont susceptibles d'agir sans bruit et d'influencer l'offre de traitement et ses modalités de réalisation.

Afin de poursuivre cet objectif, nous nous servirons de trois termes pour qualifier le but inconscient ultime d'une intervention psychothérapique en milieu correctionnel (ou de réadaptation) : guérir, soigner, aider. En partant de ces trois représentations de l'intervention, nous examinerons quels peuvent être les fondements inconscients potentiellement actifs chez l'intervenant.

1. Professeure à l'École de criminologie de l'Université de Montréal, Case postale 6128, Succursale Centre-ville, Montréal, Québec, H3C $3 \mathrm{~J} 7$. 


\section{L'INTERVENANT AU QUOTIDIEN}

Le clinicien œuvrant en centre d'accueil de réadaptation ou en milieu carcéral occupe une position fort inconfortable. Messager et porte-parole de l'institution qui l'engage, il doit gérer un dossier, récompenser et punir, transmettre des décisions sans appel et formuler des recommandations dont les conséquences peuvent être déterminantes pour l'individu concerné. Or, nous savons aussi que cet intervenant est devant un être humain qui peut être retors ou souffrant et très souvent les deux à tour de rôle. Devant le versant retors, la réponse humaine du clinicien est de réagir, peut-être par la punition; devant le versant souffrant, la réponse humaine de l'intervenant est de compatir, peut-être en voulant instituer une relation d'aide avec lui. Le dilemme entre punir et guérir qui se pose à un plan éthique se heurte ici à la banalité quotidienne du travail à accomplir. D'ailleurs, très souvent, le clinicien choisira son intervention sans trop y réfléchir, bousculé par le temps et les contraintes inhérentes à ses fonctions.

Le dilemme qui consisterait à choisir entre une fonction coercitive et une fonction thérapeutique se pose ainsi fréquemment à l'intervenant criminologique et peut parfois même l'inhiber dans sa tâche. En outre, ce dilemme semble se répéter si fréquemment que le désir de conjuguer traitement et contrainte $d^{\prime} y$ participer devient une solution tentante. Cependant forcer le sujet à être aidé, qu'il le désire ou non, ou plutôt qu'il reconnaisse ce désir ou non, ne résout en fait nullement le conflit et quelle que soit sa solution à ce dilemme, l'intervenant ne peut manquer de se sentir troublé par la détresse psychologique de l'enfant, de l'adolescent ou de l'adulte sous sa charge.

En effet, tant les descriptions cliniques des enfants et adolescents placés en centres de réadaptation que les études récentes ${ }^{2}$ sur les populations adultes des milieux carcéraux démontrent très clairement comment la présence de détresse psychologique et de maladies mentales sévères y est particulièrement élevée. L'éducateur en milieu rééducatif et le criminologue clinicien en milieu carcéral connaissent mieux que tout autre cette facette de la réalité institutionnelle, et nous suggérons que c'est davantage face à cette détresse qu'il se sent sollicité que devant le dilemme éthique que suppose l'offre de service dans un contexte de contrainte. Ainsi, ce contact quotidien avec la détresse psychologique des jeunes et des détenus n'est pas sans effet sur l'intervenant et nous suggérons qu'elle le sollicite non seulement à un niveau conscient mais aussi inconscient. C'est de cette influence inconsciente qu'il sera question dans cet essai en ce que celle-ci

2. Voir Sheilagh Hodgins (1994) pour une recension récente de ces données empiriques chez les adultes incarcérés. Voir également Fréchette et LeBlanc (1987) pour une description empirique nuancée du portrait psychologique présenté par l'adolescent dit délinquant. 
plus souvent qu'autrement risque de guider l'action et les recommandations du clinicien. Nous tenterons ainsi de distinguer cette influence inconsciente selon trois finalités thérapeutiques hypothétiques : guérir, soigner et aider. En cherchant à isoler les mouvements inconscients qui peuvent influencer l'intervenant à agir cliniquement, nous espérons éclaircir les pièges inhérents à l'intervention offerte en miljeu correctionnel et réadaptatif.

\section{GUÉRIR}

Il n'est pas rare d'entendre un clinicien d'expérience en milieu carcéral ou réadaptatif parler des désillusions qui ont marqué son cheminement professionnel. Quoique certains deviennent désabusés et découragés devant l'absence de changement observé chez les enfants, adolescents ou adultes sous leur responsabilité, d'autres intervenants parleront davantage d'une étape nécessaire de désillusionnement menant à une meilleure compréhension de leur rôle, soulignant qu'ils reconnaissent désormais le caractère irréaliste des idéaux qu'ils se fixaient pour les individus sous leur charge dans leur travail clinique d'aidants. Une humilité nouvelle caractérise alors ces intervenants, et peut-être serions-nous tentés de dire qu'ils témoignent dorénavant d'un plus grand respect pour l'individualité des personnes auprès desquelles ils interviennent.

Comment expliquer ces changements de perspectives? Nous suggerons, pour tenter de les comprendre, de porter notre regard sur différentes identifications inconscientes qui peuvent animer le clinicien ainsi que sur les pièges que comporte chacune d'entre elles.

Le désabusement, la démotivation constituent peut-être des étapes nécessaires pour qui inconsciemment s'est identifié à la société dans son travail clinique. La société figure ici pour le pôle «bon» d'un clivage effectué entre le délinquant ou le criminel inconsciemment vu comme le " méchant», celui qui doit changer pour le bien de tous. Même si l'idéal du clinicien est, à première vue, noble, cet idéal repose sur une dichotomie tranchée : d'un côté les bons, ceux qui respectent la loi, qui ne se détruisent pas ou qui ne causent pas de torts aux autres et, de l'autre côté, tous ceux qui agissent contre ces principes. L'individu placé ou incarcéré est alors inconsciemment réduit à ses gestes par le clinicien. Son individualité se confond avec son comportement délinquant ou criminel pour ce professionnel. La souffrance du sujet est certes perçue : il ne s'agit pas d'un défaut d'empathie chez l'intervenant qui s'identifie inconsciemment à la société comme victime de ce sujet, mais d'une difficulté à se permettre de reconnaître la détresse psychologique du sujet sans remettre en cause le clivage inconscient qui influence sa perception de son rôle et sa vision du sujet qu'il traite. 
Paradoxalement à l'idéal élevé de ce clinicien qui ne désire rien de moins que la guérison du sujet, son identification inconsciente à la société comme victime du sujet auprès duquel il intervient l'amènera à avoir besoin de changer celui-ci, de gré ou de force. D'où la situation désespérante et le désabusement notés chez certains cliniciens lorsque leur enthousiasme thérapeutique se confronte avec la difficulté, voire l'impossibilité de satisfaire à un tel idéal thérapeutique. Malgré leurs efforts et leur investissement dans le traitement du sujet, la guérison de celui-ci ne survient pas. En effet, tant que le clivage déformant demeure en place sur la scène interne, le clinicien n'aura d'yeux que pour les manifestations du sujet qui ne correspondront pas à une norme généralisable qui serait indiscutablement du côté du «bon ». Étalon devant lequel aucun humain ne trouverait grâce ultimement, puisque fondamentalement existent en chaque être humain des affects, des fantasmes, des émotions, des pulsions agressives, hostiles, destructrices, en un mot, du «méchant » voisinant en plus ou moins grande conflictualité avec des éléments de nature plus libidinale, ressentis subjectivement comme du «bon».

Ce clivage qui marque l'idéal thérapeutique de certains intervenants survient peut-être surtout en début de carrière. L'utilisation inconsciente de ce mécanisme de défense amène celui-ci malgré lui à vouloir guérir l'autre pour protéger la société à laquelle il s'identifie sur la scène interne. Cependant, c'est pour ne pas s'identifier à un côté mauvais massivement associé au sujet que l'intervenant clinique a recours à un tel clivage. Tout se passe comme si le clinicien voulait guérir l'autre pour mieux s'en protéger, pour ne pas ressentir en lui-mêne quelque vestige de cette «méchanceté » dont il attribue inconsciemment toute la propriété au jeune ou au détenu qu'il essaie de traiter.

Les pièges d'une telle identification inconsciente sont nombreux, car en s'identifiant à la société victime du sujet, il peut devenir tentant d'imposer abusivement le changement à ce dernier. Un cas de figure extrême d'une telle identification défensive inconsciente à la société-victime peut être imaginé dans le cas de nombreux traitements dispensés par des personnes ayant présenté des symptômes ou une problématique semblables à ceux présentés par l'individu traité. L'ex-toxicomane qui épouse une philosophie de traitement telle que celle prônée par le Daytop Village et endossée par des centres para-correctionnels comme Le Portage, par exemple, peut paradoxalement devenir extrêmement sadique envers le sujet qu'il traite. Alors que le but recherché est de guérir le toxicomane, ce qui semble être visé inconsciemment est de détruire en lui tout ce qui est associé pour le thérapeute au «mal» dont il se sent lui-même porteur et quj est massivement projeté sur le sujet. Le besoin de nier en soi la présence de "mauvais" prime les considérations thérapeutiques ou éthiques. Le sujet souffrant n'existe plus dans ces cas extrêmes pour le thérapeute. L'individu en traitement 
est réduit à une représentation du mal dont il faut éliminer les moindres traces cachées. Il n'est pas fortuit que de telles entreprises thérapeutiques ressemblent tant, en pratique, à des cérémonies d'exorcisme dont le but est de faire sortir littéralement le diable du sujet, de libérer les « Forces du Bien » en contraignant les «Forces du Mal » à quitter l'âme du possédé.

Il n'est pas surprenant non plus, quoique malheureux et désolant, qu'au terme de traitements menés sous le joug de telles identifications défensives inconscientes, le sujet ressemble souvent à un adepte de secte chez qui les attitudes, le comportement et le discours apparaissent stéréotypés, peu naturels et prévisibles. En somme, pour survivre psychiquement au traitement et «guérir ", selon la volonté du thérapeute, le sujet doit renoncer à de larges pans de son individualité et accepter d'être tel que son thérapeute se le représente.

Un exemple moins extrême mais possiblement plus habituel est rencontré chez certains intervenants qui s'identifient massivement aux valeurs véhiculés par l'institution qui les engagent. Le désir de "guérir » le sujet passe cependant davantage chez ceux-ci à travers un effort de convaincre le sujet par la séduction que par la contrainte sadique. Un tel scénario inconscient semble survenir plus souvent dans un contexte institutionnel de réadaptation où l'enfant et l'adolescent sont, du fait même de leur placement, vus comme étant implicitement «en traitement». Ainsi, dans certains cas, l'éducateur, afin de se protéger du conflit qu'éveillerait en lui la reconnaissance de ses propres tendances violentes inconscientes, cherche à séduire ni plus ni moins le jeune sous sa charge afin d'amener le sujet à se modeler à une image idéalisée de lui-même, de l'institution et de la société qu'il représente. Ce faisant, l'intervenant répond à une motivation inconsciente qui vise à se protéger lui-même, grâce au clivage, de son identification profonde à la délinquance du sujet. L'idéal thérapeutique de guérir le sujet remplit alors une fonction inconsciente défensive protégeant l'intervenant contre un sentiment angoissant de se reconnaître en quelque sorte dans des attitudes, des affects, des angoisses et des comportements qu'il préfère, pour des raisons d'équilibre personnel, attribuer exclusivement au sujet. Quoique moins extrême et investi de moins de sadisme que l'exemple précédent, l'intervenant qui désire inconsciemment guérir le jeune en le séduisant et en le convainquant par la force de son charisme et de son prestige personnel de changer de comportements et d'attitude ne peut que se heurter tôt ou tard à l'échec de son entreprise thérapeutique. Lorsque le jeune ne se soumet pas, l'éducateur ne peut souvent qu'éprouver la résistance au changement de celui-ci comme un refus hostile à son égard. L'intervenant animé d'une telle motivation inconsciente est mal préparé et peu disposé à accueillir les résistances du sujet et se sentira souvent blessé narcissiquement par les attitudes de retrait ou de distanciation de celui-ci. Ainsi confronté, l'éducateur pourra être tenté de considérer le jeune comme 
réfractaire au traitement, renforcissant le clivage initial qui associe le comportement délinquant du jeune au «méchant» à combattre. Ou encore, l'intervenant pourra se mettre à douter de sa capacité d'aider des jeunes, devenir désabusé et, souvent, se sentir surtout trahi par celui ou ceux à qui il a eu le sentiment de tout donner, ou plus précisément d'offrir le «meilleur » de lui-même, c'est-à-dire cette part clivée et idéalisée offerte comme modèle identificatoire.

\section{SOIGNER}

En suggérant que l'intervenant clinique qui veut guérir le sujet s'identifie inconsciemment à la société comme victime afin de se protéger d'une identification inconsciente encore plus troublante où il reconnaîtrait en lui-même quelque chose du «mauvais » ou du «méchant» qu'il a projeté et clivé sur le délinquant ou le détenu, il devient assez clair que le travail thérapeutique effectué, quelles qu'en soient les modalités, est voué à un certain échec. En effet, il est difficile d'imaginer que dans un tel contexte, la problématique conflictuelle personnelle du sujet, sa détresse et sa souffrance intimes ne passeront pas au second rang, peut-être même loin derrière le besoin inconsciemment éprouvé par l'intervenant d'effacer toute trace de ce «mauvais» qui lui fait si peur.

Cependant, il serait abusif de proposer qu'un tel type d'identification inconsciente soit présente chez tous les intervenants cliniques ni qu'elle soit active en permanence chez ceux-ci. Ainsi, d'autres mouvements identificatoires peuvent se manifester, donnant lieu à des offres de traitement différentes et comportant des pièges thérapeutiques particuliers.

Tout comme le clinicien peut s'identifier inconsciemment à la société comme victime d'un sujet vu comme mauvais, inversement il peut s'identifier inconsciemment à la souffrance intérieure du sujet. La perspective du clinicien est ici différente, il est attentif à la détresse et porte son regard davantage sur un état subjectivement ressenti par l'autre que sur sa symptomatologie ou encore sur ses comportements et attitudes. L'intervenant mû par une telle identification inconsciente désire soigner l'autre; son souci sera de soulager sa souffrance. Cependant, en s'identifiant inconsciemment trop massivement à la souffrance intérieure identifiée chez le sujet, l'intervenant risque de confondre la part de détresse qu'il perçoit chez le sujet avec la totalité de son être, banalisant les faits, gestes ou attitudes autres du sujet qui pourtant font tout autant partie de lui.

La polémique qui refait périodiquement surface entre «comprendre » le jeune ou le détenu et le punir prend possiblement appui, pour ce qui en est du «comprendre ", du côté d'une identification inconsciente au sujet non seulement vu comme un être souffrant, mais surtout vu comme une victime. Le sens du clivage effectué par l'intervenant est ici inversé : le Moi 
du délinquant ou du détenu est inconsciemment associé aux éléments «bons » et les éléments « mauvais » sont projetés sur sa famille, l'institution qui l'héberge, bref la société dont il serait une victime innocente.

Le cas de figure caricatural est ici constitué par le psychanalyste des années 1960 qui offre une cure classique à un détenu sans égard à la demande du sujet: «Puisque je vous vois comme souffrant, vous pourrez m'utiliser et vous servir de ce traitement pour compenser un tort qui vous a été infligé » semble être l'offre implicite résultant d'une telle identification inconsciente au sujet comme victime.

Une illustration plus actuelle des avatars d'une telle identification inconsciente au sujet comme victime serait l'incapacité pour un intervenant de définir et de délimiter le cadre de son intervention; son incapacité à refuser quoi que ce soit à un jeune ou un adulte en serait une autre manifestation. Certains mouvements de demande de pardon pour des détenus incarcérés pour des crimes graves soutenus par des membres influents de la société - pensons à Norman Mailer qui parrainait l'écrivain Jack Henry Abbott incarcéré pour meurtre - se base vraisemblablement sur une telle identification inconsciente défensive où le «bon" est clivé et vu comme appartenant au Moi du détenu et le «mauvais» projeté sur la société ou ses représentants institutionnels qui sont vus comme perpétrant le tort infligé au sujet.

Derrière cette identification défensive clivée où le «bon » est mis dans le détenu et le «mauvais » du côté de la société, se cache une identification inconsciente encore plus troublante pour l'intervenant. Il s'agit d'une identification inconsciente aux éléments violents, destructeurs devant lesquels l'intervenant est contraint soit de chercher à s'en débarrasser en les projetant massivement sur la société, auquel cas il lui devient difficile de travailler au sein d'une de ses institutions, soit encore d'accepter cette identification aux éléments violents qui serviront alors à nourrir un sentiment inconscient de culpabilité qui le tenaillera et l'amène à croire qu'il n'en fait jamais assez pour le sujet.

Une telle identification semble également étayer la conviction chez certains intervenants cliniques que tout délinquant, tout détenu doit recevoir une aide psychothérapique, quel que soit le besoin exprimé ou vaguement manifesté par le sujet. L'intervenant se place cependant alors inconsciemment dans la position d'un représentant «bon » de la société qui a le devoir de réparer les torts causés à un innocent, tort dont il se sent inconsciemment coupable. La relation d'aide qu'il cherchera alors à instaurer avec chaque individu sous sa charge constitue en quelque sorte le paiement de sa "dette " personnelle et n'est pas nécessairement en accord avec le besoin ou le désir du sujet de participer à une telle relation d'aide. 
En se plaçant ainsi, tout se passe comme si l'intervenant s'identifiait inconsciemment à la société (famille, école, institution) vue comme mauvaise. Un sentiment de culpabilité apparaît alors avoir infiltré son offre thérapeutique et nuire à son jugement clinique. En effet, mû par de telles identifications inconscientes, le clinicien peut difficilement distinguer le besoin du sujet de son propre besoin de le soigner et peut-être, peut-on ajouter, de son besoin de soulager sa propre culpabilité. En effet, l'identification au sujet comme victime protège l'intervenant d'une identification inconsciente plus fondamentale dans laquelle il se voit comme agressif et hostile, ce qui éveille et nourrit en lui un sentiment troublant de culpabilité. En ce sens, le risque d'échec de l'entreprise thérapeutique devient grand. En outre, une certaine confusion de rôles menace l'intervenant clinique qui effectue une telle identification inconsciente dès que ses fonctions comportent un volet l'associant à l'autorité, car l'exercice de toute forme d'autorité Jui fait craindre d'être envahi par son sentiment intime de culpabilité.

Ainsi, une telle identification défensive au sujet vu comme une victime peut renvoyer inconsciemment l'intervenant au sentiment d'être secrètement mauvais et, de ce fait, d'être directement en cause dans la souffrance du sujet. Le défaut de réussir à soulager la souffrance de celui-ci devient, ainsi, extrêmement désespérant pour l'intervenant qui se sent confronté à un sentiment décourageant d'impuissance et d'incapacité. En effet, s'il ne peut soulager la souffrance du sujet, le soigner, il se sent inconsciemment confronté à cette part «mauvaise » de lui-même, clivée et projetée sur la famille, l'institution, la société mais dont il ne sait que faire. La recherche effectuée par Lalande (1990) semble décrire phénoménologiquement un tel scénario identificatoire, alors que les intervenants semblent s'identifier inconsciemment au détenu comme victime et désirer ainsi d'abord et avant tout établir une relation d'aide avec ce dernier qui s'avère non seulement incompatible avec les exigences de leur fonction mais, en outre, très souvent non sollicitée par le détenu. Ainsi, l'identification au détenu comme une victime révèle inévitablement la seconde face du clivage qui consiste à attribuer le rôle de "méchant » sinon à la société, du moins au "système » pris dans son sens large et indiscriminé. Inconsciemment, l'intervenant cherche cependant à se protéger par une telle identification défensive de la part hostile, violente et haineuse en lui-même que sa relation avec les détenus lui rappelle. En reportant celle-ci sur l'institution ou le système pénal en bloc, pourrait-on dire, il se protège d'un conflit interne inévitable pour quiconque désire offrir une aide psychologique à autrui. La relation d'aide offerte par l'intervenant aux prises avec une telle motivation inconsciente et un tel désir de soigner le détenu risque donc d'être unidirectionnelle et de donner lieu à un désabusement à moyen ou à long terme après une phase où l'intervenant a eu l'impression de s'être épuisé à soulager une détresse sans cesse renouvelée chez le sujet qu'il désire soigner. 


\section{AIDER}

Peut-être le terme d'aider serait-il celui qui pourrait le mieux représenter une certaine réalité de l'intervention clinique en milieu correctionnel. Aider dans son sens de favoriser, épauler, soutenir l'autre qui le demande. Si les identifications inconscientes dont il a été question jusqu'à maintenant se caractérisent par leurs aspects conflictuels et défensifs et, tout particulièrement, par le recours à un clivage entre «bon » et «mauvais », tout mouvement identificatoire inconscient ne donne pas nécessairement lieu à pareilles stratégies défensives. Ainsi, l'empathie, caractéristique psychologique nécessaire pour qui est mû par le désir d'aider, est basée sur un mouvement identificatoire inconscient où l'on reconnaît chez l'autre quelque chose que l'on a déjà entrevu en soi. En effet, on ne peut être empathique et comprendre ce qu'il y a de nouveau, d'inconnu et d'étranger en l'autre que si cet inconnu en lui nous est déjà un tant soit peu connu par notre expérience personnelle intime.

Ainsi, le risque encouru dans toute entreprise thérapeutique est de voir en l'autre davantage ce qu'on y a mis que ce qu'il y a de singulier ou de propre au sujet. Aussi, quiconque se sert de ses capacités d'empathie comme outil de travail est susceptible de connaître des dérapages et des butées, étant donné la nature essentiellement inconsciente de cet outil qu'est l'empathie. Dans un certain sens, il peut être dit que le terme aider renvoie à tous les efforts que l'intervenant doit faire pour se questionner sur ses propres motivations cachées inhérentes à toute offre thérapeutique. Aider pourrait être associé à l'expression populaire "faire son possible ", entendant par là la reconnaissance que l'intervenant errera sûrement, se trompera assurément mais qu'en dermière analyse, il n'aura pas transformé sa tentative de venir en aide au jeune ou au détenu en un processus d'assujettissement de celui-ci.

Ainsi la question de savoir quelle aide psychothérapique spécialisée peut-on vraisemblablement offrir au jeune en institution ou à l'adulte détenu sans tomber dans les pièges identificatoires esquissés par les termes de guérir et de soigner demeure sans réponse univoque. Probablement aucun modẻle psychothérapique spécifique ne serait-il à ériger en norme ni à rejeter en bloc. En effet, les différents modèles répondent à des conceptualisations théoriques distinctes et visent des objectifs de traitement particuliers. En ce sens, aucun modèle ne peut prétendre à l'heure actuelle constituer l'outil thérapeutique de choix pour l'ensemble de la population placée ou incarcérée ni être vu comme permettant au clinicien d'éviter les pièges identificatoires décrits.

La tâche de l'intervenant clinique qui cherche à aider l'individu qu'il reçoit dans le cadre de ses fonctions l'amène donc à devoir se situer par rapport à une pluralité d'approches dont il devra connaître les fondements 
théoriques, les modalités d'application et les indications spécifiques. Une telle formation lui permettra d'être outillé de façon suffisamment nuancée pour pouvoir évaluer les besoins du sujet et l'orienter adéquatement sans présupposer d'emblée que celui-ci désire nécessairement nouer une relation d'aide ou s'engager dans un processus psychothérapique.

Or, cette nécessaire tâche évaluative accomplie, que se passe-t-il sur la scène interne au niveau des identifications inconscientes chez l'intervenant qui ne vise qu'à aider le sujet dont il a la responsabilité ? Il est possible d'imaginer qu'un jeu complexe d'identifications inconscientes est sollicité. La différence essentielle repose probablement sur le degré de liberté relative dont jouit l'intervenant lorsque son jugement clinique n'est pas trop influencé par une identification inconsciente défensive massive soit à la victime, soit à la société. En effet, quiconque désire aider un autre doit pouvoir s'identifier à la souffrance et à la détresse de celui-ci. Toutefois, si un tel mouvement identificatoire inconscient est massif, comme il a été illustré à l'aide des représentations de guérir et de soigner, et qu'il est traversé de motivations essentiellement défensives, l'intervenant perd toute sa liberté de réflexion face au sujet lui demandant de l'aide. Ce n'est dorénavant plus le besoin individuel du sujet qui guidera la recommandation de traitement ou le choix d'une approche thérapeutique, mais davantage le lien identificatoire inconscient que l'intervenant crée avec le sujet et qui en tant que source potentielle d'angoisse pour lui suscite inévitablement le besoin de s'en protéger.

En acceptant en soi-même les manifestations de différents mouvements identificatoires, en s'interrogeant et se réinterrogeant sur ses motivations profondes, en cherchant toujours à étayer ses décisions cliniques sur une réflexion qui se méfie des dogmes et des idées reçues, l'intervenant criminologique réussira probablement à mieux éviter les pièges inhérents à toute offre thérapeutique. En milieu correctionnel et rééducatif, les enjeux sont d'autant plus grands que l'intervenant clinique est souvent placé devant une exigence de réussite de ses interventions de la part de ses pairs et de l'institution. Attente d'autant plus irréaliste que les motivations à demander de l'aide de la part d'un détenu ou d'un jeune sont plus souvent qu'autrement complexes et également très souvent infiltrées là aussi d'une attente de réussite explicitement ou implicitement exigée.

\section{CONCLUSION}

À l'aide de trois termes usuels, nous avons cherché à faire figure et à permettre au lecteur de mieux se représenter les enjeux identificatoires complexes qui peuvent favoriser ou paralyser le travail psychothérapique en milieu correctionnel. Devant l'importance de la souffrance humaine et de la détresse psychologique rencontrées par l'intervenant clinique tant en 
centre d'accueil pour enfants et adolescents qu'en milieu carcéral, il importe de ne pas banaliser la tâche complexe qui est celle de l'intervenant sollicité par deux maîtres potentiels qui s'opposent.

Pour l'intervenant clinique dont les fonctions l'amènent à être fortement impliqué dans le quotidien du sujet, le paradigme identificatoire conscient le plus susceptible de le guider est probablement celui de la fonction parentale. Dans sa fonction parentale, en effet, l'adulte se place comme le principal responsable du développement de son enfant; il aura tantôt à l'encadrer ou à lui imposer son autorité, tantôt à le soutenir, à comprendre ses difficultés et l'aider à les surmonter. Sans sombrer dans un paternalisme ou une séduction de l'autre qui puisse brimer sa liberté d'être et de penser, ce modèle est dans un certain sens valable non seulement pour l'éducateur qui travaille auprès d'enfants et d'adolescents, mais aussi pour l'intervenant travaillant auprès d'adultes incarcérés. Élaborer ce point de vue et l'argumenter de façon suffisante exigeraient cependant un développement qui dépasse le cadre de notre propos.

Dans ce sens, pour le clinicien cuvrant en milieu réadaptatif ou correctionnel, la voie la plus sûre est de tenter de s'appuyer sur le besoin manifesté par l'individu et implique une nécessaire étape évaluative afin que l'offre thérapeutique qui sera faite au sujet non seulement corresponde à des besoins singuliers mais de plus réponde à une motivation personnelle chez lui à recevoir de l'aide. Cependant, ni le criminologue clinicien ni l'éducateur ne peuvent espérer remplir ces fonctions de façon nuancée dans l'isolement. Ce sont, en effet, lorsque les conditions de travail encouragent le repli sur soi, découragent les initiatives de formation continue, minimisent les occasions de discussions de cas entre professionnels pairs et ne favorisent pas la possibilité de recevoir de la supervision non autoritaire, non institutionnellement hiérarchisée, que le passage à l'acte d'identifications inconscientes défensives massives est le plus susceptible de survenir. Et cela au détriment non seulement du jeune placé ou de l'adulte incarcéré, mais tout autant de l'intervenant clinique qui tôt ou tard risque le désceuvrement, la démotivation ou une fermeture narcissique défensive qui le contraindront à la suffisance ou à l'isolement. 


\section{BIBLIOGRAPHIE}

BERTRAND, M. A. (1981), «Réflexions critiques sur l'intervention et les limites du droit à intervenir », Santé mentale au Québec, vol. VI, $\mathrm{n}^{\circ} 2$, novembre, pp. 5-11.

FRÉCHETTE, M. et LEBLANC, M. (1987), Délinquances et délinquants, Montréal, Gaétan Morin.

HODGINS, S. (1994), « Les malades mentaux face à la justice criminelle », in D. Szabo et M. LeBlanc, (dir.), Traité de criminologie empirique, $2^{e}$ édition, Montréal, PUM, pp. 323-350.

LALANDE, P. (1990), "Comment devient-on "réaliste". Une étude sur la trajectoire mentale des agents de probation ", Déviance et société, vol. 14, $\mathrm{n}^{\circ} \mathrm{l}$, pp. 17-38.

MILL, J. S. (1970), On Liberty (1859) in Essential Works of J.S. Mill, New York, Bantam. 